\title{
Potential drug interactions in patients with rheumatoid arthritis
}

\author{
Fabíola Bagatini ${ }^{1}$, Carine Raquel Blatt ${ }^{2}$, Gabriela Maliska ${ }^{3}$, Gunter Voges Trespash ${ }^{3}$, \\ Ivânio Alves Pereira ${ }^{4}$, Adriana Fontes Zimmermann ${ }^{5}$, Bernd Heinrich Storb ${ }^{6}$, Mareni Rocha Farias ${ }^{7}$
}

\begin{abstract}
Introduction: The term polypharmacy, meaning the concomitant use of multiple medications by one individual, has been widely reported in institutionalized or elderly patients. It can, however, occur in patients with chronic diseases, such as rheumatoid arthritis (RA). Objective: To quantify polypharmacy in a group of RA patients and to assess the risk of potential undesirable interactions between medications used for managing RA and those used for non-chronic diseases. Methods: A cohort study was carried out with 103 RA patients registered at the Strategy of Access to Medications from the Brazilian Health Ministry, at the School of Pharmacy of the city of Florianópolis, state of Santa Catarina. Patients were monthly followed up by use of form completion. Drug interactions were identified by use of the Drugdex System - Thomson Micromedex ${ }^{\circledR}$ - Interactions database. Results: Polypharmacy was found in $95.1 \%$ of the patients, and 19 potential undesirable interactions were observed between the drugs used by 74 patients (mean of $3.0 \pm 1.2$ interactions/ patient). All potential interactions were related to methotrexate. Omeprazole was the major representative, accounting for $29.3 \%$ of the interactions, followed by diclofenac sodium (17.6\%), and metamizole sodium (13.2\%). Conclusion: Considering that this study confirms that polypharmacy is a common therapeutic practice in RA patients, it is worth emphasizing the need for greater surveillance regarding the adverse effects or effectiveness reduction of certain drugs due to drug interaction.
\end{abstract}

Keywords: rheumatoid arthritis, drug interaction, polypharmacy, antirheumatic drugs.

[Rev Bras Reumatol 2011;51(1):20-39] CElsevier Editora Ltda.

\section{INTRODUCTION}

Rheumatoid arthritis (RA) is a chronic inflammatory autoimmune disease, characterized by progressive symmetric polyarticular impairment, and, in some cases, extra-articular manifestations. ${ }^{1,2}$

Rheumatoid arthritis is estimated to affect $0.5 \%$ to $1 \%$ of the world population, women being three to four times more affected than men. ${ }^{3,4}$

Ideal therapy varies according to the patient's individual characteristics, such as disease stage, activity, and severity, in addition to the response to previous treatment regimens. ${ }^{5,6}$
Currently, the following five classes of drugs that benefit RA patients are available: analgesics; non-steroidal antiinflammatory drugs (NSAID); corticosteroids; disease modifying anti-rheumatic drugs (DMARD); and biological drugs.

As the prevalence of comorbidities and risk factors, such as dyslipidemias, diabetes mellitus, hypertension, obesity, and osteoporosis, increases with age, ${ }^{7}$ the prescription of concurrent medications is frequent in RA patients. In addition, the use of medications for symptomatic treatments and/or self-medication can further increase the number of drugs used by RA patients.

Polypharmacy deserves special attention, because medications are chemical substances that can interact with each

\footnotetext{
Submitted on 06/7/2010. Approved on 12/16/2010. We declare no conflict of interest. Ethics Committee n. 193296.

Universidade Federal de Santa Catarina - UFSC, Brazil.

1. Pharmacist - UFSC

2. Pharmacist - M.Sc., UFSC

3. Undergraduate student in Pharmacy, UFSC; Pharmacy Professor of UniSul

4. Chief physician of the Service of Rheumatology, UFSC - Doctor in Rheumatology, USP

5. Assistant physician of the Service of Rheumatology, UFSC - Candidate for Doctor's Degree in Medical Sciences, UFSC

6. Collaborative researcher - Doctor in Production Engineering, UFSC

7. Pharmacist, Professor of the Post-Graduation Program in Pharmacy, UFSC - Doctor in Natural Sciences by the Pharmazeutisches Institut - Universitat Bonn, Germany

Correspondence to: Mareni Rocha Farias. Universidade Federal de Santa Catarina. Departamento de Ciências Farmacêuticas. Campus Universitário, Trindade. CEP: 88040-970. Florianópolis, SC, Brazil. Phone.: +55 48 3721-9567. Email: marenif@yahoo.com.br
} 
other, with nutrients, or with environmental chemical agents and trigger undesirable or iatrogenic responses. ${ }^{8}{ }^{89}$ Despite the lack of consensus about what number of medications expresses polypharmacy, many authors define it as the association of six or more medications or as the administration of a greater number of medications than that clinically indicated. ${ }^{10,11}$

The term polypharmacy has been associated with institutionalized and elderly patients, but it can occur in other groups of patients with chronic diseases, such as RA. Thus, this study aimed at quantifying polypharmacy in RA patients and at assessing the risk of potential undesirable interactions between medications used for managing RA and those used for treating non-chronic affections.

\section{METHODOLOGY}

Our data were obtained from a cohort study of RA patients registered at the Strategy of Access to Medications from the Brazilian Health Ministry, at the School of Pharmacy of the UFSC/PMF, in the city of Florianópolis, state of Santa Catarina, from August 2008 to February 2010.

The sample comprised adult RA patients of both sexes, living in the city of Florianópolis and registered at the Strategy of Access to Medications from the Brazilian Health Ministry to receive the following medications: adalimumab, infliximab, etanercept (biologic agents), and leflunomide (DMARD).

Patients who did not want or could not participate in the study were excluded (20), and the following patients were considered as losses: those who did not sign the written informed consent (4); those who abandoned the study (8); and a deceased patient (1). The total sample comprised 103 patients. Total follow-up included one month of initial assessment and monthly follow-up for 12 months.

Data were collected by the major author during monthly interviews with patients, at the occasion of drug dispensation, by using previously validated follow-up forms. The following variables were collected: sex, age, medications used for RA treatment, and other medications used during the study period with or without medical prescription.

Considering the maximum number of drug associations used, the patients were classified according to the presence or absence of polypharmacy. In this study, polypharmacy was defined as the association of six or more medications, regardless of the duration. Topical medications, domestic formulations, and eye solutions were not included in the evaluation.

This study assessed the existence of possible interactions between the medications used for treating non-chronic affections and those used for treating RA. The medications used for treating non-chronic affections were those used by patients at any time of the study and that had no indication to be used in chronic diseases. The drugs used in the symptomatic RA treatment were included in the group of drugs for nonchronic affections.

Those medications used for non-chronic diseases during the follow-up of the RA patients were classified according to the Anatomical Therapeutic Chemical (ATC) Classification ${ }^{12}$ into their therapeutic groups.

The ATC Classification is an international classification recommended by the World Health Organization. It consists in classifying medications into different groups and subgroups (levels) according to the organ or system upon which the medications act and according to their chemical, pharmacological, and therapeutic properties. There are five levels, the fifth level being the active chemical substance. ${ }^{12}$

The Drugdex System - Thomson Micromedex ${ }^{\circledR}$ Interactions ${ }^{13}$ database was used to assess reports of possible interactions between the drugs previously cited.

An association of the presence of potential interactions was observed with the variables sex, age bracket, presence of polypharmacy, and therapeutic schemes including methotrexate, by using the chi-square $\left(\chi^{2}\right)$ and Fisher's Exact tests, with $95 \%$ confidence interval.

The associations showing potential drug interaction were classified and presented according to their severity, scientific evidence available, and time for the beginning of the adverse effect, according to that same database.

Regarding severity, the drug associations were classified as follows: contra-indicated, when the concomitant administration of drugs is not recommended; major, when the association represents a threat to life, requiring immediate medical intervention; moderate, when the patient's clinical findings get worse, requiring alteration of the medicamentous therapy instituted; minor, when the patient's clinical findings change, but alterations in the medicamentous therapy are not required. ${ }^{13}$

The scientific evidence available about drug interactions was classified as follows: excellent, when controlled clinical trials confirm the existence of drug interaction; good, when documents about the interaction exist, but controlled clinical trials lack; fair, when documents about the interaction are scarce, but pharmacological considerations of the occurrence of the interaction exist; unknown, when no document in the literature confirms the drug interaction. ${ }^{13}$

Regarding the time for the beginning of adverse effects, interactions were classified as follows: rapid, when the adverse effects resulting from the interaction occur within less than 24 hours; slow, when the adverse effects resulting 
from the interaction do not occur within the first 24 hours; nonspecified, when there is no report in the literature about the time for the beginning of adverse effects after the simultaneous administration of the drugs. ${ }^{13}$

This study was approved by the Committee on Ethics in Research with Human Beings of the Universidade Federal de Santa Catarina (protocol 103/2008).

\section{RESULTS}

Regarding the sample, the female sex represented $89.7 \%$ of the patients, and the age ranged from 22 to 83 years (median, 57.4 years; mean, $56.9 \pm 13.1$ years).

Figure 1 shows the therapeutic regimens used by patients on study occasions $\mathrm{T} 0, \mathrm{~T} 4, \mathrm{~T} 8$, and $\mathrm{T} 12$, corresponding to $0,4,8$, and 12 months, respectively. The combinations of DMARDs $(49 \%, 44 \%, 39 \%$, and $36 \%$ at T0, T4, T8, and T12, respectively) and of DMARDs and biological agents (35\%, $37 \%, 35 \%$, and $35 \%$ at $\mathrm{T} 0, \mathrm{~T} 4, \mathrm{~T} 8$, and $\mathrm{T} 12$, respectively) predominated, and methotrexate was present in approximately $60 \%$ of the therapeutic regimens. Throughout the study, a reduction in the association of DMARDs and an increase in the use of a biological agent in isolation (from 3\% at T0 to $8 \%$ at $\mathrm{T} 12$ ) and of a DMARD in isolation (from $15 \%$ at $\mathrm{T} 0$ to $24 \%$ at T12) were observed.

The association of leflunomide and methotrexate was the predominant therapeutic regimen throughout the study.

The presence of polypharmacy was observed in $95.1 \%$ of the patients (Table 1). The means of the minimum and maximum numbers of medications associated per patient were $7.5 \pm 3.2$ and $12.2 \pm 4.1$, respectively.

Of the medications used by the patients studied, only those for treating non-chronic diseases were selected for assessing the risk of drug interaction, and they corresponded to 105 different active substances of 19 therapeutic groups.

Figure 2 shows the percentage frequency of the therapeutic groups used in the RA patients for treating their non-chronic diseases during the period studied. The three most frequently used therapeutic groups were the anti-inflammatory products $(17.6 \%)$, analgesics (17.6\%), and corticosteroids (15.8\%). In such groups, the most used drugs were diclofenac sodium $(36.4 \%)$, paracetamol (43.2\%), and prednisone $(72.3 \%)$, respectively.

The literature consulted evidenced 19 potential interactions, all related to methotrexate (Chart 1). Drug associations with potential interactions were used by 74 patients, in a total of 205 potential interactions and mean of $3.0 \pm 1.2$ potential interactions per patient. Nine patients showed only one potential interaction (Chart 2).
The literature also shows three potential drug interactions referring to chloroquine diphosphate and four others related to methotrexate, but no patient studied reported the use of such associations.

Of the variables assessed, only the presence of polypharmacy showed a positive correlation with the existence of drug interactions (Table 2).

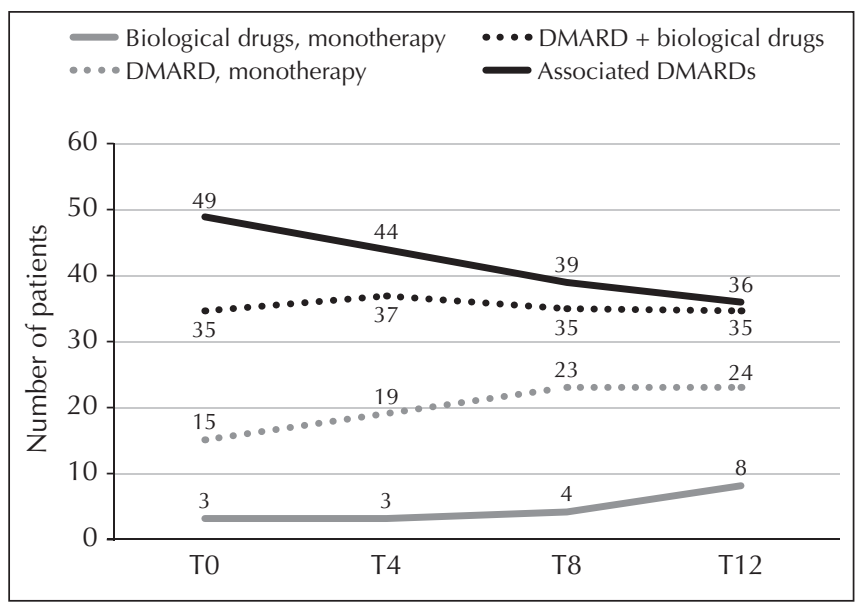

Figure 1

Therapeutic regimens used by patients undergoing treatment for RA.

\section{Table 1}

Presence of polypharmacy, means of the minimum and maximum numbers of medications used by patients undergoing treatment for RA.

\begin{tabular}{lc}
\hline Variables & Frequency or Mean \pm SD* $^{*}$ \\
\hline Total number of medications used & 1,836 \\
$\begin{array}{l}\text { Mean of the total number of } \\
\text { medications used per patient }\end{array}$ & $17.8 \pm 6.9$ \\
$\begin{array}{l}\text { Mean of the minimum number of } \\
\text { medications associated per patient }\end{array}$ & $7.5 \pm 3.2$ \\
$\begin{array}{l}\text { Mean of the maximum number of } \\
\text { medications associated per patient }\end{array}$ & $12.2 \pm 4.1$ \\
Presence of polypharmacy & $\%(\mathbf{n})$ \\
Yes & $95.1 \%$ \\
Male & $9.2 \%(9)$ \\
Female & $90.8 \%(89)$ \\
No & $4.9 \%$ \\
Male & $20.0 \%(1)$ \\
Female & $80.0 \%(4)$ \\
\hline
\end{tabular}

* SD: standard deviation 




Figure 2

Percentage frequency of the therapeutic groups used for treating non-chronic diseases of patients undergoing RA treatment.
Table 2

Association of the variables sex, age, presence of polypharmacy, and therapeutic regimen including methotrexate with the presence of interaction of medications used for treating non-chronic diseases and those used by patients undergoing treatment for severe RA.

\begin{tabular}{|c|c|c|c|c|}
\hline Variables & $\begin{array}{c}\text { With } \\
\text { interaction } \\
(\mathrm{N}=74)\end{array}$ & $\begin{array}{c}\text { No } \\
\text { interaction } \\
(\mathrm{N}=29)\end{array}$ & $\begin{array}{l}\text { Relative risk } \\
\left(95 \% \mathrm{Cl}^{*}\right)\end{array}$ & P-value \\
\hline \multicolumn{5}{|l|}{ Sex } \\
\hline Male & 6 & 3 & \multirow{2}{*}{$\begin{array}{c}0.92(0.57- \\
1.49)\end{array}$} & \multirow{2}{*}{0.7177} \\
\hline Female & 68 & 26 & & \\
\hline \multicolumn{5}{|c|}{ Age bracket } \\
\hline$<60$ years & 39 & 18 & \multirow{2}{*}{$\begin{array}{c}0.90(0.71- \\
1.14)\end{array}$} & \multirow{2}{*}{0.3898} \\
\hline$\geq 60$ anos & 35 & 11 & & \\
\hline \multicolumn{5}{|c|}{ Presence of polypharmacy } \\
\hline Yes & 73 & 25 & \multirow{2}{*}{$\begin{array}{c}3.72(0.64- \\
21.58)\end{array}$} & \multirow{2}{*}{0.0214} \\
\hline No & 1 & 4 & & \\
\hline \multicolumn{5}{|c|}{ Therapeutic regimen for RA including methotrexate } \\
\hline Yes & 74 & 3 & \multirow{2}{*}{ - } & \multirow{2}{*}{-} \\
\hline No & 0 & 26 & & \\
\hline
\end{tabular}

${ }^{*} \mathrm{Cl}$ : Confidence interval.

\section{Chart 1}

Distribution of medications used for non-chronic diseases according to their therapeutic group, ATC classification, number of patients, and potential interactions with medications used in the RA treatment.

\begin{tabular}{|c|c|c|c|c|c|c|c|c|c|c|c|}
\hline \multirow[b]{2}{*}{ Therapeutic group } & \multirow[b]{2}{*}{ Medications } & \multirow[b]{2}{*}{ ATC } & \multicolumn{9}{|c|}{$\begin{array}{l}\text { Number of patients using the association and } \\
\text { presence of potential interaction }\end{array}$} \\
\hline & & & LEF1 & $\mathrm{MTX}^{2}$ & $\mathrm{CQN}^{3}$ & $\mathrm{HCQ}^{4}$ & $\mathrm{ABA}^{5}$ & $\mathrm{ADA}^{6}$ & $\mathrm{ETA}^{7}$ & INF $^{8}$ & RIT $^{9}$ \\
\hline \multirow{7}{*}{$\begin{array}{l}\text { Drugs for acid- } \\
\text { related disorders }\end{array}$} & Esomeprazole magnesium & $\mathrm{A} 02 \mathrm{BC} 05$ & 4 & 3 & 0 & 0 & 0 & 1 & 0 & 2 & 0 \\
\hline & Famotidine & A02BA03 & 1 & 0 & 1 & 0 & 0 & 0 & 0 & 0 & 0 \\
\hline & Lansoprazole & A02BC03 & 1 & 2 & 0 & 0 & 0 & 1 & 0 & 0 & 0 \\
\hline & Omeprazole & A02BC01 & 54 & $60^{*}$ & 3 & 5 & 1 & 18 & 8 & 9 & 1 \\
\hline & Pantoprazole sodium & $\mathrm{A} 02 \mathrm{BC} 02$ & 2 & $3 *$ & 0 & 0 & 0 & 1 & 0 & 0 & 0 \\
\hline & Rabeprazole sodium & $\mathrm{A} 02 \mathrm{BC} 04$ & 1 & 1 & 0 & 0 & 0 & 0 & 0 & 0 & 0 \\
\hline & Ranitidine, hydrochloride & A02BA02 & 1 & 2 & 0 & 0 & 0 & 1 & 0 & 1 & 0 \\
\hline \multirow{8}{*}{$\begin{array}{l}\text { Drugs for functional } \\
\text { gastrointestinal } \\
\text { disorders }\end{array}$} & Bromopride & A03FA04 & 2 & 2 & 0 & 1 & 0 & 0 & 0 & 1 & 0 \\
\hline & Domperidone & A03FA03 & 2 & 1 & 0 & 0 & 0 & 0 & 0 & 0 & 0 \\
\hline & Scopolamine, butylbromide & A03BB01 & 3 & 8 & 0 & 0 & 0 & 3 & 0 & 1 & 0 \\
\hline & Isometheptene mucate & A03AX10 & 5 & 7 & 0 & 0 & 1 & 6 & 0 & 1 & 0 \\
\hline & Metoclopramide hydrochloride & A03FA01 & 10 & 13 & 2 & 0 & 1 & 5 & 0 & 2 & 0 \\
\hline & Octylonium bromide & A03AB06 & 1 & 0 & 0 & 0 & 0 & 0 & 0 & 0 & 0 \\
\hline & Papaverine hydrochloride & A03AD01 & 2 & 2 & 0 & 0 & 0 & 0 & 0 & 0 & 0 \\
\hline & Pinaverium bromide & A03AX04 & 1 & 1 & 0 & 0 & 0 & 0 & 0 & 1 & 0 \\
\hline \multirow{2}{*}{$\begin{array}{l}\text { Antiemetics and } \\
\text { antinauseants }\end{array}$} & Dimenhydrinate & A04AD & 2 & 3 & 0 & 0 & 0 & 1 & 0 & 0 & 0 \\
\hline & Ondansetron hydrochloride & A04AA01 & 3 & 4 & 1 & 0 & 0 & 1 & 0 & 2 & 0 \\
\hline
\end{tabular}




\begin{tabular}{|c|c|c|c|c|c|c|c|c|c|c|c|}
\hline \multirow{3}{*}{$\begin{array}{l}\text { Antidiarrheals, } \\
\text { intestinal anti- } \\
\text { inflammatory/anti- } \\
\text { infective agents }\end{array}$} & Loperamide hydrochloride & A07DA03 & 1 & 0 & 0 & 0 & 0 & 0 & 0 & 0 & 0 \\
\hline & Nystatin & A07AA02 & 3 & 3 & 0 & 0 & 0 & 0 & 0 & 0 & 0 \\
\hline & Racecadotril & A07XA04 & 0 & 1 & 0 & 0 & 0 & 1 & 0 & 0 & 0 \\
\hline Urological drugs & Phenazopyridine hydrochloride & G04BX06 & 2 & 0 & 0 & 1 & 0 & 0 & 0 & 0 & 0 \\
\hline \multirow{6}{*}{ Corticosteroids } & Betamethasone & H02АB01 & 9 & 8 & 0 & 0 & 0 & 5 & 0 & 1 & 0 \\
\hline & Deflazacort & H02AB13 & 6 & 4 & 0 & 0 & 0 & 1 & 0 & 2 & 0 \\
\hline & Dexamethasone & $\mathrm{H} 02 \mathrm{AB} 02$ & 4 & 4 & 1 & 0 & 0 & 0 & 0 & 1 & 0 \\
\hline & Prednisolone & H02АB06 & 4 & 6 & 0 & 1 & 0 & 3 & 1 & 0 & 0 \\
\hline & Prednisone & $\mathrm{H} 02 \mathrm{AB} 07$ & 62 & 65 & 5 & 6 & 3 & 19 & 7 & 11 & 1 \\
\hline & Triamcinolone acetonide & H02АВ08 & 0 & 1 & 1 & 0 & 0 & 0 & 0 & 0 & 0 \\
\hline \multirow{22}{*}{ Antibacterials } & Amoxicillin & J01CA04 & 10 & $14^{*}$ & 0 & & 0 & 2 & 3 & 2 & 0 \\
\hline & Amoxicillin + potassium clavulanate & J01CE30 & 5 & $3^{*}$ & 0 & 0 & 0 & 0 & 0 & 1 & 0 \\
\hline & Dihydrate azithromycin & J01FA10 & 14 & 10 & 1 & 0 & 0 & 2 & 2 & 2 & 0 \\
\hline & Benzathine benzylpenicillin & J01CE08 & 1 & $2^{*}$ & 0 & 0 & 0 & 1 & 0 & 0 & 0 \\
\hline & Cefalexin monohydrate & J01DB01 & 8 & 8 & 0 & 0 & 0 & 2 & 1 & 0 & 0 \\
\hline & Cefepime hydrochloride & J01DE01 & 1 & 1 & 0 & 0 & 0 & 0 & 0 & 0 & 0 \\
\hline & Ceftriaxone disodium & J01DD04 & 1 & 1 & 0 & 0 & 0 & 0 & 0 & 0 & 0 \\
\hline & Cefuroxime sodium & J01DC02 & 2 & 1 & 0 & 0 & 0 & 0 & 0 & 0 & 0 \\
\hline & Ciprofloxacin hydrochloride & J01MA02 & 13 & $19^{*}$ & $0^{*}$ & 2 & 1 & 5 & 2 & 5 & 1 \\
\hline & Clarithromycin & J01FA09 & 2 & 2 & $0^{*}$ & 0 & 0 & 0 & 0 & 0 & 0 \\
\hline & Doxycycline hydrochloride & J01AA02 & 1 & $1^{*}$ & 0 & 0 & 0 & 0 & 0 & 0 & 0 \\
\hline & Fosfomycin trometamol & J01XX01 & 0 & 1 & 0 & 0 & 0 & 1 & 0 & 0 & 0 \\
\hline & Gentamicin sulfate & J01GB03 & 1 & 1 & 0 & 0 & 0 & 0 & 0 & 0 & 0 \\
\hline & Levofloxacin hemihydrate & J01MA12 & 7 & 5 & 0 & 1 & 0 & 0 & 0 & 3 & 0 \\
\hline & Metronidazole & J01XD01 & 2 & 1 & 0 & 0 & 0 & 0 & 0 & 0 & 0 \\
\hline & Minocycline hydrochoride & J01AA08 & 1 & 0 & 0 & 0 & 0 & 0 & 0 & 0 & 0 \\
\hline & Moxifloxacin hydrochloride & J01MA14 & 2 & 1 & 0 & 0 & 0 & 0 & 0 & 0 & 0 \\
\hline & Nitrofurantoin & J01XE01 & 4 & 3 & 0 & 0 & 0 & 0 & 0 & 0 & 0 \\
\hline & $\begin{array}{l}\text { Nitrofurantoin }+ \\
\text { sulfamethoxypyridazine }+ \\
\text { phenylzodiamine piridine }\end{array}$ & J01ED20 & 1 & 0 & 0 & 1 & 0 & 0 & 0 & 0 & 0 \\
\hline & Norfloxacin & J01MA06 & 9 & 4 & 1 & 0 & 1 & 0 & 0 & 0 & 0 \\
\hline & Sulfamethoxazole trimethoprim & J01EE01 & 3 & $1^{*}$ & 0 & 2 & 0 & 1 & 0 & 0 & 0 \\
\hline & Tetracycline hydrochloride & J01AA07 & 0 & $1 *$ & 0 & 0 & 0 & 0 & 0 & 1 & 0 \\
\hline \multirow{2}{*}{ Antimycotics } & Ketoconazole & J02AB02 & 1 & 1 & 0 & 0 & 0 & 0 & 0 & 0 & 0 \\
\hline & Fluconazole & J02AC01 & 5 & 3 & $0^{*}$ & 0 & 0 & 2 & 0 & 1 & 0 \\
\hline \multirow{2}{*}{ Antivirals } & Aciclovir & J05AB01 & 1 & 1 & 0 & 0 & 0 & 0 & 0 & 1 & 0 \\
\hline & Oseltamivir phosphate & J05AH02 & 1 & 1 & 0 & 0 & 0 & 0 & 0 & 0 & 0 \\
\hline \multirow{12}{*}{$\begin{array}{l}\text { Anti-inflamatory } \\
\text { products }\end{array}$} & Celecoxib & M01AH01 & 2 & 2 & 0 & 1 & 0 & 1 & 0 & 0 & 0 \\
\hline & Ketoprofen & M01AE03 & 5 & $3^{*}$ & 1 & 0 & 0 & 1 & 0 & 0 & 0 \\
\hline & $\begin{array}{l}\text { Chondroitin sulfate + } \\
\text { glucosamine sulfate }\end{array}$ & M01AX25 & 3 & 0 & 1 & 0 & 0 & 1 & 0 & 0 & 0 \\
\hline & Diclofenac potassium & M01AB05 & 1 & $0^{*}$ & 0 & 0 & 0 & 0 & 0 & 0 & 0 \\
\hline & Diclofenac sodium & M01AB05 & 32 & $36^{*}$ & 2 & 3 & 1 & 12 & 3 & 10 & 1 \\
\hline & Etodolac & M01AB08 & 1 & $1^{*}$ & 0 & 0 & 0 & 0 & 0 & 1 & 0 \\
\hline & Etoricoxib & M01AH05 & 3 & 1 & 0 & 0 & 0 & 1 & 0 & 0 & 0 \\
\hline & Phenylbutazone calcium & M01AA01 & 2 & $1^{*}$ & 0 & 0 & 0 & 0 & 0 & 0 & 0 \\
\hline & Ibuprofen & M01AE01 & 6 & $4^{*}$ & 3 & 0 & 0 & 2 & 1 & 0 & 0 \\
\hline & Mefenamic acid & M01AG01 & 2 & $0^{*}$ & 0 & 0 & 0 & 0 & 0 & 0 & 0 \\
\hline & Meloxicam & M01AC06 & 5 & 6 & 3 & 0 & 0 & 1 & 1 & 2 & 0 \\
\hline & Naproxen sodium & M01AE02 & 3 & $3^{*}$ & 0 & 0 & 1 & 2 & 0 & 0 & 0 \\
\hline \multirow{3}{*}{$\begin{array}{l}\text { Anti-inflamatory } \\
\text { products }\end{array}$} & Nimesulide & M01AX17 & 27 & $22^{*}$ & 3 & 1 & 0 & 2 & 3 & 3 & 0 \\
\hline & Piroxicam & M01AC01 & 1 & $\mathbf{0}^{*}$ & 1 & 0 & 0 & 0 & 0 & 0 & 0 \\
\hline & Tenoxicam & M01AC02 & 3 & $\mathbf{0}^{*}$ & 1 & 0 & 0 & 0 & 0 & 0 & 0 \\
\hline Muscle relaxants & Carisoprodol & M03BA02 & 9 & 6 & 2 & 1 & 0 & 2 & 1 & 1 & 0 \\
\hline
\end{tabular}




\begin{tabular}{|c|c|c|c|c|c|c|c|c|c|c|c|}
\hline \multicolumn{12}{|c|}{$\nabla$} \\
\hline \multirow{2}{*}{ Muscle relaxants } & Cyclobenzaprine hydrochloride & М03ВХ08 & 5 & 3 & 2 & 0 & 0 & 0 & 0 & 0 & 0 \\
\hline & Orphenadrine citrate & M03BC51 & 5 & 4 & 0 & 0 & 0 & 1 & 0 & 0 & 0 \\
\hline \multirow{8}{*}{ Analgesics } & Acetylsalicylic acid & N02BA01 & 5 & $3^{*}$ & 0 & 0 & 0 & 0 & 0 & 1 & 0 \\
\hline & Lysine clonixinate & N02BG61 & 1 & 1 & 0 & 0 & 0 & 0 & 0 & 0 & 0 \\
\hline & Codein phosphate & N02AA08 & 12 & 8 & 1 & 1 & 0 & 2 & 1 & 4 & 0 \\
\hline & Dihydroergotamine mesylate & N02CA01 & 2 & 0 & 1 & 0 & 0 & 0 & 1 & 0 & 0 \\
\hline & Metamizole sodium & N02BB02 & 28 & $27^{*}$ & 0 & 1 & 2 & 11 & 2 & 4 & 0 \\
\hline & Naratriptan hydrochloride & N02CC02 & 1 & 1 & 0 & 1 & 0 & 0 & 0 & 0 & 0 \\
\hline & Paracetamol & N02BE01 & 38 & 38 & 4 & 5 & 1 & 10 & 5 & 11 & 0 \\
\hline & Tramadol hydrochloride & N02AX02 & 3 & 2 & 1 & 0 & 0 & 0 & 0 & 2 & 0 \\
\hline Psycholeptics & Hydroxyzine hydrochloride & N05BB01 & 1 & 2 & 1 & 0 & 0 & 2 & 0 & 1 & 0 \\
\hline Antiprotozoals & Secnidazole & P01AB07 & 1 & 0 & 0 & 0 & 0 & 0 & 0 & 0 & 0 \\
\hline \multirow{2}{*}{ Anthelmintics } & Albendazole & P02CA03 & 3 & 1 & 0 & 0 & 0 & 2 & 0 & 0 & 0 \\
\hline & Ivermectin & P02CF01 & 4 & 3 & 0 & 0 & 0 & 2 & 0 & 0 & 0 \\
\hline \multirow{3}{*}{ Nasal preparations } & Oxymetazoline hydrochloride & R01AA05 & 1 & 0 & 0 & 0 & 0 & 0 & 0 & 0 & 0 \\
\hline & Phenylephrine hydrochloride & R01BA03 & 5 & 1 & 2 & 0 & 0 & 0 & 0 & 0 & 0 \\
\hline & Pseudoephedrine sulfate & R01BA03 & 1 & 1 & 0 & 1 & 0 & 0 & 1 & 0 & 0 \\
\hline \multirow{9}{*}{$\begin{array}{l}\text { Drugs for obstructive } \\
\text { airway diseases }\end{array}$} & Acebrophylline & R03DA & 1 & 1 & 0 & 0 & 0 & 0 & 0 & 0 & 0 \\
\hline & Beclomethasone dipropionate & R03BA01 & 2 & 2 & 0 & 0 & 0 & 0 & 0 & 0 & 0 \\
\hline & Budesonide & R03BA02 & 4 & 2 & 0 & 0 & 0 & 0 & 0 & 0 & 0 \\
\hline & Phenoterol hydrobromide & R03AC04 & 2 & 2 & 0 & 0 & 0 & 0 & 0 & 0 & 0 \\
\hline & Fluticasone furoate & R03BA05 & 1 & 0 & 0 & 0 & 0 & 0 & 0 & 0 & 0 \\
\hline & Formoterol fumarate & R03AC13 & 1 & 0 & 0 & 0 & 0 & 0 & 0 & 0 & 0 \\
\hline & Ipratropium bromide & R03BB01 & 3 & 2 & 0 & 0 & 0 & 0 & 0 & 0 & 0 \\
\hline & Salbutamol sulfate & R03AC02 & 2 & 2 & 0 & 0 & 0 & 0 & 0 & 0 & 0 \\
\hline & Theophylline & R03DA04 & 1 & $1 *$ & 0 & 0 & 0 & 0 & 0 & 0 & 0 \\
\hline \multirow{2}{*}{$\begin{array}{l}\text { Cough and cold } \\
\text { preparations }\end{array}$} & Acetylcysteine & R05CB01 & 2 & 3 & 0 & 0 & 0 & 0 & 1 & 0 & 0 \\
\hline & Ambroxol & R05CB06 & 1 & 0 & 0 & 0 & 0 & 0 & 0 & 0 & 0 \\
\hline \multirow{8}{*}{ Antihistamines } & Carbinoxamine maleate & R06AA08 & 1 & 0 & 1 & 0 & 0 & 0 & 0 & 0 & 0 \\
\hline & Chlorpheniramine maleate & R06AB04 & 7 & 5 & 1 & 0 & 1 & 1 & 1 & 0 & 0 \\
\hline & Desloratadine & R06AX27 & 1 & 0 & 0 & 0 & 0 & 0 & 0 & 0 & 0 \\
\hline & Dexchlorpheniramine maleate & R06AB02 & 2 & 1 & 0 & 0 & 0 & 0 & 0 & 0 & 0 \\
\hline & Epinastine hydrochloride & R06AX24 & 0 & 0 & 0 & 0 & 0 & 0 & 0 & 1 & 0 \\
\hline & Fexofenadine hydrochloride & R06AX26 & 1 & 2 & 0 & 0 & 0 & 0 & 0 & 1 & 0 \\
\hline & Loratadine & R06AX13 & 3 & 2 & 1 & 0 & 0 & 0 & 0 & 1 & 0 \\
\hline & Promethazine hydrochloride & R06AD02 & 1 & 1 & 0 & 0 & 0 & 0 & 0 & 0 & 0 \\
\hline
\end{tabular}

${ }^{1}$ LEF: Leflunomide; ${ }^{2} \mathrm{MTX}$ : Methotrexate; ${ }^{3} \mathrm{CQN}$ : Chloroquine diphosphate; ${ }^{4 H C Q: ~ H y d r o x y c h l o r o q u i n e ~ s u l f a t e ; ~}{ }^{5} \mathrm{ABA}$ : Abatacept; ${ }^{\mathrm{A} A D A}$ : Adalimumab; ${ }^{7} \mathrm{ETA}:$ Etanercept; ${ }^{8} \mathrm{NNF}$ : Infliximab; ${ }^{9} \mathrm{RIT}$ : Rituximab * Existence of potential drug interaction reports.

Omeprazole was the major representative of the risk for potential drug interaction identified in the study, corresponding to $29.3 \%$ of them, followed by diclofenac sodium (17.6\%), and metamizole sodium (13.2\%).

Regarding the potential interactions identified in the study, $78.9 \%$ were classified as major, $21.0 \%$ had fair scientific evidence, and, in $21.0 \%$, the beginning of adverse effects was rapid (Chart 2).

\section{DISCUSSION}

This study aimed at providing information about the risk of potential drug interactions in RA patients, considering that, in Brazil, that type of information is rare.
Studies have shown that combinations of DMARDs are more effective than monotherapy. ${ }^{14,15,16}$ In addition, biological agents are more effective when combined with DMARDs, in particular methotrexate. ${ }^{17,18,19}$ This justifies the strategy of combining DMARDs or biological agents found in most patients in this study.

The means of the minimum and maximum numbers of medications used per patient in this study were $7.5 \pm 3.2$ and $12.2 \pm 4.1$, respectively. In a survey about medications used by retired elderly, the mean number of medications used was 4.1 $\pm 3.0 .{ }^{20}$ In 2006, Loyola Filho et al.,${ }^{21}$ studying the prevalence of medication consumption in 1,598 elderly, reported that the mean number of medications used was 2.18 . 


\section{Chart 2}

Distribution of medications reported as having a potential interaction with methotrexate according to the number of users, severity of the interaction, scientific evidence available, and time for the beginning of adverse effects. $\mathrm{N}=103$ patients.

\begin{tabular}{|c|c|c|c|c|}
\hline Medications & $\begin{array}{l}\text { Number of patients using } \\
\text { the MTX association }{ }^{1}\end{array}$ & Severity & Evidence & $\begin{array}{l}\text { Time for the beginning } \\
\text { of adverse effects }\end{array}$ \\
\hline Acetylsalicylic acid & 3 & Major & Good & Rapid \\
\hline Amoxicillin & 14 & Major & Good & Slow \\
\hline Amoxicillin + potassium clavulanate & 3 & Major & Good & Slow \\
\hline Benzathine benzylpenicillin & 2 & Major & Good & Slow \\
\hline Ketoprofen & 3 & Major & Good & Slow \\
\hline Diclofenac sodium & 36 & Major & Good & Slow \\
\hline Metamizole sodium & 27 & Major & Good & Slow \\
\hline Doxycycline hydrochloride & 1 & Major & Good & Rapid \\
\hline Etodolac & 1 & Major & Fair & Slow \\
\hline Phenylbutazone calcium & 1 & Major & Fair & Slow \\
\hline Ibuprofen & 4 & Major & Good & Slow \\
\hline Naproxen sodium & 3 & Major & Good & Slow \\
\hline Nimesulide & 22 & Major & Good & Slow \\
\hline Omeprazole & 60 & Major & Good & Rapid \\
\hline Sulfamethoxazole + Trimethoprim & 1 & Major & Excellent & Slow \\
\hline Ciprofloxacin hydrochloride & 19 & Moderate & Fair & $\mathrm{NS}^{2}$ \\
\hline Pantoprazole sodium & 3 & Moderate & Good & Rapid \\
\hline Tetracycline hydrochloride & 1 & Moderate & Fair & $\mathrm{NS}^{2}$ \\
\hline Theophylline & 1 & Moderate & Good & Slow \\
\hline
\end{tabular}

'MTX: Methotrexate, ${ }^{2}$ NS: Noy specified.

The mean number of medications used and the frequency of polypharmacy in our study were higher than those found in other national studies. ${ }^{22,23}$ However, studies about medication use and the term polypharmacy are usually associated with institutionalized and/or elderly patients, hindering the comparison of the results obtained with those of other studies.

According to Prybys et al. ${ }^{24}$, in elderly patients, the risk of adverse effects, including drug interactions, increases $13 \%$ with the use of two medications, $58 \%$ with the use of five medications, reaching $82 \%$ in the presence of seven or more medications.

Polypharmacy is associated with an increase in the risk and severity of adverse reactions, in the risk of triggering drug interactions, of causing cumulative toxicity and mistakes during medication administration, and of reducing adherence to treatment. Therefore, it is directly related to health care costs, which include medications and the repercussions of their use, such as costs of visits to specialists, emergency health care, and hospital admission. ${ }^{22,25}$
In this study, the most frequently used therapeutic groups were the anti-inflammatory products $(17.6 \%)$, analgesics (17.6\%), and corticosteroids (15.8\%). These groups and their representatives are used for treating RA, and, in more severe cases, their full doses can be administered, ${ }^{6,26}$ which explains their greater frequency of use.

It is worth noting that, in this study, the detection of potential drug interactions was based in an information technology tool included in the Drugdex System - Thomson Micromedex ${ }^{\circledR}$ - Interactions ${ }^{13}$ database, which cannot consider aspects related to patients, dosages, and sequence and time of medication administration. This, thus, may overestimate the incidence and risk of potential drug interactions.

All potential drug interactions found in this study were related to methotrexate. In the literature consulted, no potential interaction of the medications selected in this study with those used for RA management (abatacept, adalimumab, etanercept, infliximab, leflunomide, and hydroxychloroquine sulfate) has been reported. . $^{13,27,28}$ 
Methotrexate is the most used initial therapy in RA patients, except for those with liver disease. ${ }^{26}$ Despite its excellent efficacy and tolerability profiles, in addition to its low cost, ${ }^{29}$ methotrexate can interact with other medications, resulting in toxicity.

In this study, 19 types of potential interactions with methotrexate were observed, 15 of which were classified as major and four as moderate. This emphasizes the need for being cautious when prescribing and dispensing other medications to patients already using methotrexate in their treatment regimens, as well as the need for instructing patients regarding self-medication.

The interactions related to methotrexate and classified as major can result in an increase in the serum concentrations of its active metabolite, potentiating its adverse effects, such as leukopenia, thrombocytopenia, anemia, liver toxicity, nephrotoxicity, and mucosal ulcerations. ${ }^{1,13,27,28}$

An increase in the serum concentrations of methotrexate, with signs and symptoms of toxicity, was observed when the drug was coadministered with a variety of penicillins, such as amoxicillin, benzylpenicillin, mezlocillin, piperacillin..$^{30,31}$ Because of the structural similarity between penicillins and methotrexate, a competitive inhibition of methotrexate tubular secretion can occur, increasing its half-life. ${ }^{28,32,33}$

According to the Drugdex System - Thomson Micromedex ${ }^{\circledR}$ - Interactions ${ }^{13}$ database, tetracyclines and other oral antibiotics can reduce the effectiveness of methotrexate by reducing its intestinal absorption or interfering with its enteropathic circulation, inhibiting the intestinal flora and suppressing the drug metabolism by bacteria. However, other authors believe that tetracyclines and their derivatives increase methotrexate serum concentrations. . $^{27,34,35}$

The association of high doses of methotrexate with doxycycline can result in gastrointestinal and hematologic toxicity. ${ }^{34}$ The interaction mechanism remains unknown, but it is believed to be related to the removal of methotrexate from its binding sites in serum proteins, the competition for renal tubular secretion, or the inhibition of the renal synthesis of prostaglandins. ${ }^{13,27,34,35}$

No potential interaction between minocycline and methotrexate has been reported, but as minocycline is a tetracycline derivative, its association with methotrexate should be monitored.

Pancytopenia and myelotoxicity have been reported after the concomitant administration of methotrexate and sulfamethoxazole + trimethoprim..$^{36,37,38}$ The literature suggests the following two mechanisms of interaction: 1) sulfonamides can remove methotrexate from its binding site to serum proteins or reduce its renal excretion; and 2) additive inhibition of dihydrofolate reductase by methotrexate and trimethoprim. ${ }^{36,39}$

In addition to pancytopenia and myelotoxicity, that association can increase the risk of megaloblastic anemia because methotrexate and sulfonamides can produce a folate deficiency by suppressing dihydrofolate reductase.,13,27,28

The concomitant use of methotrexate and ciprofloxacin can result in an increase in the serum concentrations of the former due to the inhibition of its renal tubular transportation caused by the latter.,13,27,28 Two cases of severe toxicity have been reported, and, thus, the association of high doses of methotrexate and ciprofloxacin should be avoided. ${ }^{40}$

The association of methotrexate and NSAIDs can cause several complications, such as severe hematologic and gastrointestinal toxicity. ${ }^{13,35}$ In addition, preexisting renal dysfunctions (or NSAID-induced renal dysfunctions) potentiate the risk for adverse reactions. ${ }^{13,27,28}$ That interaction can occur through four different mechanisms: 1) competition between methotrexate and NSAIDs for renal tubular secretion; 2) removal of methotrexate, or its active metabolite, from the binding site to serum proteins; 3 ) a reduction in the liver metabolism of methotrexate caused by NSAIDs; and 4) NSAIDs inhibit the synthesis of prostaglandins (vasoconstrictors of renal capillaries), which results in a decrease in renal blood flow, and, thus, in the glomerular filtration of methotrexate. ${ }^{41,42}$

Some studies have reported that the coadministration of methotrexate, piroxicam, naproxen, ketoprofen, and ibuprofen in RA patients did not affect the pharmacokinetic profile of methotrexate. ${ }^{43,44}$

Although the concomitant administration of NSAIDs and methotrexate can potentially cause severe toxicity, low doses of that association are considered well tolerated, but the appearance of severe adverse effects should always be cautiously monitored. ${ }^{13,35}$

Although metamizole sodium and acetylsalicylic acid have been classified as analgesics according to the ATC Classification, in the literature consulted, their mechanisms of interaction with methotrexate have been compared with the general mechanism of NSAIDs. . $3,27,28,45,46$

The major representative of the potential drug interactions identified was omeprazole, accounting for $29.3 \%$ of them. Its interaction was classified as major and the beginning of adverse effects was rapid. The concomitant use of methotrexate and omeprazole can increase the risk of toxicity of the former, ${ }^{13,27,28,47}$ because, according to Suzuki et al. ${ }^{48}$ the coadministration of proton pump inhibitors can delay the excretion of methotrexate and 
potentiate its adverse effects.Omeprazole serum concentrations decrease rapidly after interruption of its use. . $3,27,28,47$ Thus, patients receiving that association should be strictly monitored to avoid possible damage resulting from the high concentration of methotrexate in their bodies. ${ }^{13,27,28}$

The interaction between pantoprazole and methotrexate is less severe due to the degree of activation of pantoprazole according to the $\mathrm{pH}$ of the medium. With a $\mathrm{pH}$ of approximately 5 , as that found in the renal tubules, pantoprazole is less active than omeprazole, having, thus, a lower inhibitory effect on methotrexate tubular secretion. ${ }^{49}$

A double-blind, placebo-controlled study with 15 adult asthmatic patients was carried out to assess the effects of the concomitant use of theophylline and methotrexate..$^{50}$ Eight patients received methotrexate and seven received placebo for six weeks. After six weeks, theophylline excretion reduced from 48 to $38.9 \mathrm{~mL} / \mathrm{h} / \mathrm{kg}$ in patients treated with methotrexate. The serum concentrations of theophylline should be monitored when methotrexate is introduced, discontinued, or has its dosage altered. ${ }^{13,50}$

Patients with RA undergo treatment protocols with multiple medications and for prolonged time. Those are important factors, which, when combined, can contribute to the occurrence of adverse reactions and undesirable drug interactions, which can worsen the pathophysiological scenario already installed and/or require the suspension or change of the therapeutic regimen.

It is worth emphasizing the need for assessing the riskbenefit relation of each drug association, as well as the adoption of measures that can reduce negative effects, such as changing the time of drug administration and monitoring adverse effects related to drug association.

The practice of self-medication, mainly for pain control, is common among RA patients, which puts them at greater risk for drug interactions, adverse effects, and toxicity. The therapeutic group with the highest potential of interaction with the medications used for RA treatment was that of the anti-inflammatory drugs.

To minimize or prevent the potential risk of adverse reactions and drug interactions, contributing to the rational use of medications and improvement of the health conditions of RA patients, the following is recommended: multidisciplinary teams; pharmacotherapeutic follow-up; and education of patients regarding the practice of self-medication. 


\section{REFERENCES}

\section{REFERÊNCIAS}

1. Pinto MRC, Miguel RCC, Rezende GG. Rheumatoid arthritis treatment. Rev Bras Reumatol 2006; 46(3):219-23.

2. Venables PJW, Maini RN. Clinical features of rheumatoid arthritis. In: Greene JM, editor. UpToDate $161^{\text {a }}$ ed. Maini RN: UpToDate; 2008.

3. Senna ER, De Barros AL, Silva EO, Costa IF, Pereira LV, Ciconelli RM et al. Prevalence of rheumatic diseases in Brazil: a study using the COPCORD approach. J Rheumatol 2004; 31:594-7.

4. Organização Mundial de Saúde (OMS), 2009. Disponível em http:// wwwwhoint/chp/topics/rheumatic/en/. [Acesso em 28 de agosto de 2009].

5. Harris Jr ED. Clinical Features of Rheumatoid Arthritis. In: Harris Jr ED, Budd RC, Firestein GS, Genovese MC, Sergent JS, Ruddy S et $a l$, editors. Kelleys Textbook of Rheumatology. $7^{\text {th }}$ ed. Philadelphia: Elsevier-Saunders 2005; 1043-100.

6. BRASIL. Portaria SCTIE $n^{\circ} 66$ de 06 de novembro de 2006. Aprova o Protocolo Clínico e Diretrizes Terapêuticas - AR. Diário Oficial da União, Poder Executivo, Brasília, DF, 06 de novembro de 2006.

7. Torigoe DY, Laurindo IMM. Artrite Reumatóide e Doenças Cardiovasculares Rev Bras Reum 2006; 46(1):60-6.

8. Secoli SR. Interações medicamentosas: fundamentos para a prática clínica da enfermagem. Rev Esc Enfermagem USP 2001; 35(1):26-32.

9. Souza PM, Santos-Neto LL, Kusano LTE, Pereira MG. Diagnóstico e controle da polifarmácia no idoso. Rev Saúde Pública 2007; 41(6):1049-53.

10. Steinman MA, Rosenthal GE, Landefeld CS, Bertenthal D, Sen S, Kaboli PJ. Conflicts and concordance between measures of medication prescribing quality. Med Care 2007; 45(1):95-9. 
11. Bushardt RL, Massey EB, Simpson TW, Ariail JC, Simpson KN. Polypharmacy: misleading, but manageable. Clin Interv Aging 2008; 3(2):383-9.

12. Organização Mundial de Saúde (OMS), 2010. WHO Collaborating Centre for Drug Statistics Methodology. Disponível em http://www. whocc.no/atc_ddd_index. [Acesso em 10 de janeiro de 2010].

13. Drugdex System. Greenwood Village: Thomson Micromedex ${ }^{\mathbb{B}}$ Healthcare Series 20: Interactions, 2010. Disponível em http:// periodicoscapesgovbr. [Acesso em 28 de janeiro de 2010].

14. Pincus T, O'dell JR, Kremer JM. Combination therapy with multiple disease-modifying antirheumatic drugs in rheumatoid arthritis: a preventive strategy. Ann Intern Med 1999; 131(10):768-74.

15. Gabriel SE, Tugwell P, Drummond M. Progress towards an OMERACT-ILAR guideline for economic evaluations in rheumatology. Ann Rheum Dis 2002; 61:370-3.

16. Lee SS, Park YW, Park JJ, Kang YM, Nam EJ, Kim SI et al. Combination treatment with leflunomide and methotrexate for patients with active rheumatoid arthritis. Scand J Rheumatol 2009; 38(1):11-14.

17. Weinblatt ME, Keystone EC, Furst DE, Moreland LW, Weisman MH, Birbara CA et al. Adalimumab, a fully human anti-tumor necrosis factor alpha monoclonal antibody, for the treatment of rheumatoid arthritis in patients taking concomitant methotrexate: the ARMADA trial. Arthritis Rheum 2003; 48(1):35-45

18. Breedveld FC, Weisman MH, Kavanaugh AF, Cohen SB, Pavelka $\mathrm{K}$, van Vollenhoven $\mathrm{R}$ et al. The PREMIER Study A multicenter, randomized, double-blind clinical trial of combination therapy with adalimumab plus methotrexate versus methotrexate alone or adalimumab alone in patients with early, aggressive rheumatoid arthritis who had not had previous methotrexate treatment. Arthritis Rheum. 2006; 54:26-37.

19. van Vollenhoven RF, Ernestam S, Geborek P, Petersson IF, Cöster $\mathrm{L}$, Waltbrand $\mathrm{E}$ et al. Addition of infliximab compared with addition of sulfasalazine and hydroxychloroquine to methotrexate in patients with early rheumatoid arthritis (Swefot trial): 1-year results of a randomised trial. Lancet 2009; 374(9688):459-66.

20. Ribeiro AQ, Rozenfeld S, Klein CH, Cesar CC, Acurcio FA. Inquérito sobre uso de medicamentos por idosos aposentados, Belo Horizonte, MG. Rev Saúde Pública 2008; 42(4):724-32.

21. Loyola-Filho AI, Uchoa E, Costa-Lima MF. Estudo epidemiológico de base populacional sobre uso de medicamentos entre idosos na Região Metropolitana de Belo Horizonte, Minas Gerais, Brasil. Cad Saúde Pública 2006; 22(12):2657-67.

22. Carvalho MFC. A Polifarmácia em idosos no município de São Paulo - Estudo SABE - Saúde, Bem-estar e Envelhecimento São Paulo - SP [dissertação de mestrado]. São Paulo: Faculdade de Saúde Pública da Universidade de São Paulo; 2007.

23. Flores LM, Mengue SS. Uso de medicamentos por idosos em região do sul do Brasil. Rev Saúde Pública 2005; 39(6):924-9.

24. Prybys KM, Melville K, Hanna J, Gee A, Chyka P. Polypharmacy in the elderly: clinical challenges in emergency practice: part 1 overview, etiology, and drug interactions. Emerg Med Rep 2002; 23(8):145-53.

25. Secoli SR. Polifarmácia: interações e reações adversas no uso de medicamentos por idosos. Rev Bras Enferm 2010; 63(1):136-40.

26. Harris DE, Schur PH, Maini RN. Overview of the management of rheumatoid arthritis. In: Rose BD, editor. UpToDate $141^{\mathrm{a}}$ ed. Wellesley: UpToDate; 2006.
27. Stockley's IH. Drug interactions. $6^{\text {a }}$ ed. London: Pharmaceutical Press; 2002.

28. Tatro DS, editor. Drug interaction facts. St. Louis: Facts and Comparisons; 2004.

29. Sokka T, Pincus T. Ascendancy of weekly low-dose methotrexate in usual care of rheumatoid arthritis from 1980 to 2004 at two sites in Finland and the United States. Rheumatology (Oxford) 2008; 47:1543-7.

30. Dean R, Nachman J, Lorenzana NA. Possible methotrexatemezlocillin interaction. Am J Pediatr Hematol Oncol 1992; 14:88-92.

31. Ronchera CL, Hernandez T, Peris JE, Torres F, Granero L, Jiménez NV et al. Pharmacokinetic interaction between high-dose methotrexate and amoxicillin. Ther Drug Monit 1993; 15:375-9.

32. Sathi N, Ackah J, Dawson J. Methotrexate induce neutropenia associated with coprescription of penicillins: serious and underreported? Rheumatology (Oxford) 2005; 44(8):1051-5.

33. Sathi N, Dawson J. Methotrexate-induced pancytopenia associated with co-prescription of penicillin and trimethoprim. Clin Rheumatol 2007; 26(1):134-5.

34. Tortajada-Ituren JJ, Ordovas-Baines JP, Liopis-Salvia P, JiménezTorres NV. High-dose methotrexate-doxycycline interaction. Ann Pharmacother 1999; 33:804-8.

35. Lacy CF, Armstrong LL, Goldman MP, Lance LL. Drug Information Handbook. 17 ed. Hudson: Lexi-Comp; 2008.

36. Thomas MH, Gutterman LA. Methotrexate toxicity in a patient receiving trimethoprim-sulfamethoxazole. J Rheumato 1986; 13:440-1.

37. Maricic M, Davis M, Gall EP. Megaloblastic pancytopenia in a patient receiving concurrent methotrexate and trimethoprimsulfamethoxazole treatment. Arthritis Rheum 1986; 29:133-5.

38. Steurer A, Gumpel JM. Methotrexate and trimethoprim: a fatal interaction (letter). Br J Rheumatol 1998; 37:105-106.

39. Groenendal H, Rampen FHJ. Methotrexate and trimethoprimsulphamethoxazole-a potentially hazardous combination. Clin Exp Dermatol 1990; 15:358-60.

40. Dalle JH, Auvrignon A, Vassal G, Leverger G. Interaction between methotrexate and ciprofloxacin. J Pediatr Hematol Oncol 2002; 24(4):321-2.

41. Chan A, Ko Y, Wong CM. Clinically Significant Drug-Drug Interactions Between Oral Anticancer Agents and Nonanticancer Agents: Profiling and Comparison of Two Drug Compendia. Ann Pharmacother 2008; 42(12):1737-48.

42. Maeda A, Tsuruoka S, Kanai Y, Endou H, Saito K, Miyamoto E et al. Evaluation of the interaction between nonsteroidal anti-inflammatory drugs and methotrexate using human organic anion transporter 3-transfected cells Eur J Pharmacol 2008; 596:166-72.

43. Tracy TS, Worster T, Bradley JD, Greene PK, Brater DC. Methotrexate disposition following concomitant administration of ketoprofen, piroxicam and flurbiprofen in patients with rheumatoid arthritis. Br J Clin Pharmacol 1994; 37:453-6.

44. Vakily M, Amer F, Kukulka MJ, Andhivarothal N. Coadministration of lansoprazole and naproxen does not affect the pharmacokinetic profile of methotrexate in adult patients with rheumatoid arthritis. J Clin Pharmacol 2005; 45(10):1179-86.

45. Furst DE, Herman RA, Koehnke R, Ericksen N, Hash L, Riggs CE et al. Effect of aspirin and sulindac on methotrexate clearance. J Pharm Sci 1990; 79:782-6. 
46. Stewart CF, Fleming RA, Germain BF, Seleznick MJ, Evans WE. Aspirin alters methotrexate disposition in rheumatoid arthritis patients. Arthritis Rheum 1991; 34:1514-20.

47. Reid T, Yuen A, Catolico M, Carlson RW. Impacto of omeprazole on the plasma clearance of methotrexate. Cancer Chemother Pharmacol 1993; 33:82-4.

48. Suzuki K, Doki K, Homma M, Tamaki H, Hori S, Ohtani H et al. Co-administration of proton pump inhibitors delays elimination of plasma methotrexate in high-dose methotrexate therapy. Br J Clin Pharmacol 2009; 67(1):44-9.
49. Beorlegui B, Aldaz A, Ortega A, Aquerreta I, Sierrasesúmega L, Giráldez J. Potential interaction between methotrexate and omeprazole. Ann Pharmacother 2000; 34(9):1024-7.

50. Glynn-Barnhart AM, Erzurum SC, Leff JA, Martin RJ, Cochran JE, Cott GR et al. Effect of low-dose methotrexate on the disposition of glucocorticoids and theophylline. J Allergy Clin Immunol 1991; $88: 180-6$ 